\title{
O uso de tecnologias leves em tempos de pandemia: Um relato de experiência
}

\author{
The use of soft technologies in times of pandemic: An experience report \\ El uso de las tecnologías blandas en tiempos de pandemia: Un informe de experiencia
}

Recebido: 02/07/2021 | Revisado: 08/07/2021 | Aceito: 12/07/2021 | Publicado: 23/07/2021

\author{
Sandra Regina Peixoto de Sousa \\ ORCID: https://orcid.org/0000-0002-4163-7660 \\ Universidade Federal Fluminense, Brasil \\ E-mail: sandreca01@gmail.com \\ Karla Maria Neves Memória Lima \\ ORCID: https://orcid.org/0000-0002-2813-6589 \\ Universidade Federal Fluminense, Brasil \\ E-mail: karla.memoria@gmail.com \\ Ândrea Cardoso de Souza \\ ORCID: https://orcid.org/0000-0002-6549-8634 \\ Universidade Federal Fluminense, Brasil \\ E-mail: andriacsouza@gmail.com
}

\begin{abstract}
Resumo
A motivação da construção desta narrativa é tecer uma reflexão sobre a execução do procedimento de punção venosa periférica, tomando como um dos fundamentos para essa realização a adoção de tecnologias leves, relacionais como dispositivo para minimizar os medos e anseios dos pacientes diante de tal procedimento. Objetivou-se descrever a experiência de uma enfermeira na adoção das tecnologias leves nos momentos da pré e pós-punção venosa periférica, em um ambulatório de oncologia, incorporando o emprego da estratégia das tecnologias leve como o uso de prática associada a um cuidado de qualidade. Relato de experiência vivida de forma mais intensa no período de pandemia, no ambulatório de oncologia de um hospital público da região sudeste do Brasil. Temos como resultado a redução da sensação dolorosa, por parte dos usuários pacientes, após a punção venosa. Concluímos que a adoção das tecnologias leves, também conhecidas como relacionais pela equipe de enfermagem, auxilia na diminuição do temor dos pacientes diante da punção venosa periférica, fato que coaduna com a adoção de boas práticas e assim contribuindo para a promoção da saúde desses pacientes.
\end{abstract}

Palavras-chave: Enfermagem; Pandemias; Serviços de saúde; Neoplasias; Promoção da saúde; Covid-19.

\begin{abstract}
The motivation of the construction of this narrative is to weave a reflection on the execution of the peripheral venous puncture procedure, taking as one of the foundations for this realization the adoption of mild, soft technologies as a device to minimize the fears and anxiety of patients before such a procedure. This study aimed to describe the experience of a nurse in the adoption of soft technologies in moments of pre and post-peripheral venous puncture, in an oncology outpatient clinic, incorporating the use of a strategy of light technologies like the use of practice associated with quality care. Relate of lived experience-reported in a more intense way during the pandemic period, in the oncology outpatient clinic of a public hospital in the southeastern region of Brazil. As a result, it was noticed the reduction of patient painful sensation after the venous puncture. We conclude that the adoption of soft technologies, also known as relational by the nursing team helps reducing fear of patients in the face of peripheral venous puncture, a fact that is consistent with the adoption of good practices and thus contributing to the promotion of these patients' health.
\end{abstract}

Keywords: Nursing; Pandemics; Health services; Neoplasms; Health promotion; Covid-19.

\section{Resumen}

La motivación de la construcción de esta narrativa es tejer una reflexión sobre la ejecución del procedimiento de punción venosa periférica, tomando como una de las bases para esta realización la adopción de tecnologías blandas y relacionales como dispositivo para minimizar los miedos y anides de los pacientes antes de procedimiento. Este estudio tuvo como objetivo describir la experiencia de un enfermero en la adopción de en los tecnologías blandas momentos de punción venosa pre y post-periférica, en un ambulatorio de oncología, incorporando el uso de la estrategia de tecnologías ligeras como el uso de la práctica asociada a la atención de calidad. Relato de experiencia vivida más intensamente en el período de pandemia, en el ambulatorio de oncología de un hospital público de la región sudeste de Brasil. Y como resultado de la reducción de la sensación dolorosa por parte de los usuarios pacientes después de la punción venosa. Concluimos que la adopción de tecnologías blandas, también conocidas como relacionales por el equipo de enfermería, ayuda a reducir el miedo de los pacientes frente a la punción venosa 
periférica, hecho que es consistente con la adopción de buenas prácticas y contribuyendo así a la promoción de la salud de esos pacientes.

Palabras clave: Enfermería; Pandemias; Servicios de salud; Neoplasias; Promoción de la salud; Covid-19.

\section{Introdução}

No mês de dezembro de 2019, na cidade de Wuhan, província de Hubei, na China, surgiu um surto de infecção, uma pneumonia causada pelo coronavírus da síndrome respiratória aguda grave 2 (SARS-Cov-2) que se espalhou em pouco tempo por muitos países, acometendo muitas pessoas. Logo que foi identificado, a Organização Mundial da Saúde (OMS), oficialmente, o denominou de coronavírus 2019 (covid-19) (Huang et al, 2019).

No Brasil, o primeiro caso foi confirmado em 12 de fevereiro 2020, e logo a doença se espalhou por todos os estados. No mês de setembro de 2020, os números oficiais do Ministério da Saúde (MS), foram: 4.657,702 de casos confirmados e 139.808 mortes pela covid-19; os quais expressam significativa alteração no registro do número de mortes e marca o ano de 2020 como um dos mais trágicos dos últimos 100 anos (Brasil, 2020).

Diante dessa situação caótica que assola o mundo, estima-se que o número de óbitos de profissionais de saúde, no boletim epidemiológico do Ministério da Saúde (número 38), foi de 305 mortes por covid-19: 100 técnicos e auxiliares de enfermagem, 60 médicos, 36 enfermeiros, além das demais categorias profissionais (Brasil, 2020).

Para que seja possível a adoção de uma assistência de qualidade, é preciso que os serviços e processos de trabalho em saúde tenham como foco os pacientes, porém, os serviços se estruturam para atender às suas próprias necessidades. Sendo assim, o usuário não é tido como o ator principal. Dessa forma, a relação enrijecida e verticalizada adotada por profissionais e serviços de saúde, sendo estes os detentores de conhecimento a respeito do processo saúde-doença em detrimento do saber do paciente, apresenta-se como um dos fatores dificultadores da adoção das tecnologias relacionais (Souza \& Souza, 2017).

O câncer já traz em si uma conotação de sofrimento e angústia. O tratamento com antineoplásicos é carregado de situações não muito agradáveis, devido, principalmente, aos efeitos colaterais que são, em sua maioria, nocivos, trazem consequências, como: náuseas e vômitos; desconforto intestinal; lesões orais; em alguns casos, erupções pelo corpo, ressecamentos e a temida queda de cabelo, entre outro. $\mathrm{O}$ medo e as incertezas relacionadas com a finitude estão presentes na maioria dos usuários que chegam neste setor. Assim, diante da situação posta pela pandemia, era preciso que a equipe de enfermagem se dedicasse ainda mais aos pacientes, não com foco centrado no procedimento, mas no paciente em sofrimento, conforme será narrado, como uma ação que busca a boa prática (Benjamin, 1987; INCA, 2015).

O objetivo deste texto, portanto, constitui narrar a experiência de uma enfermeira na adoção das tecnologias leves de cuidado à saúde nos momentos da pré e pós-punção venosa periférica como estratégia para a diminuição do medo e tensão provocados pelo procedimento nos pacientes em um ambulatório de oncologia, incorporando o emprego da estratégia das tecnologias leve como o uso de prática associada a um cuidado de qualidade e assim promover a saúde no referido ambiente.

\section{Metodologia}

Trata-se de um relato de experiência que adota a narrativa como orientação metodológica e que considera a necessidade de experimentar para narrar; desenvolvido em um ambulatório de oncologia, na sala de quimioterapia de um hospital público do estado do Rio de Janeiro no mês de setembro do ano de 2020 (Bondía, 2002; Merhy \& Franco, 2003).

A motivação da construção desta narrativa é tecer uma reflexão sobre a execução do procedimento de punção venosa periférica, tomando como um dos fundamentos para essa realização a adoção de tecnologias leves, relacionais como dispositivo para minimizar os medos e anseios dos pacientes diante de tal procedimento. (Silva \& Veras, 2016). 
Ao adotar as tecnologias leves, também conhecidas como relacionais - que são as que têm nos profissionais as principais ferramentas e que não empregam nenhum recurso tecnológico, mas sim, o acolhimento, o vínculo, a valorização do sujeito, a escuta qualificada -, a equipe de enfermagem se aproxima dos pacientes e desenvolve práticas geradoras de um melhor acolhimento, ampliando seu potencial de ação, estando em consonância com a proposta de promover uma assistência de qualidade aos pacientes, e assim também promovendo saúde segundo os princípios da Política Nacional de Promoção em Saúde (PNPS) (Brasil, 2014).

\section{Resultados e Discussão}

\subsection{O cotidiano narrado: a tensão aumentada na sala de quimioterapia na pandemia}

Durante a pandemia, a tensão dos pacientes que eram submetidos a sessões de quimioterapia na sala onde ocorria esse tratamento no ambulatório de oncologia estava intensificada, pois, além de precisarem se deslocar de suas residências, da dor do procedimento, tinham ainda que permanecer em um ambiente fechado ainda que com todos os equipamentos de proteção individual (EPI), cuidado da equipe de enfermagem, higienização adequada. Entretanto, o que fazer quando as recomendações de OMS, da Organização Pan-Americana da Saúde (Opas) e outras instituições de referência de pesquisas na saúde no país, como a Fundação Oswaldo Cruz (Fiocruz), são para evitar transporte público e locais fechados? Esse foi mais um dilema que os pacientes do ambulatório de oncologia em tratamento quimioterápico precisaram enfrentar. Então, acrescido ao temor do câncer, da dor do procedimento da punção venosa periférica, estava o da contaminação pela covid-19.

Por atuar nesse setor há mais de dois anos, ao longo desse tempo, fui identificando a necessidade de construir estratégias na minha prática que minimizassem o sofrimento com as punções decorrente do tratamento oncológico. Essa necessidade foi sendo ampliada em função da pandemia.

A construção de espaço que articule a necessidade do usuário e reduza a experiência 'dolorosa' da punção venosa periférica se constitui um cuidado que qualifica a ação de enfermagem, pois, em meu cotidiano, essa prática consiste em um recurso potente que ameniza o desconforto, a dor e a sensação de insegurança dos pacientes.

Ao chegar na sala de quimioterapia, os pacientes se vêm diante de situações não vividas e, por vezes, espantosas expectativas diante do novo. De maneira muito recorrente, eles chegam ao setor com muitas dúvidas acerca do procedimento da punção venosa periférica e apresentam questões do tipo: como será? Quantas vezes irão me furar?

Dessa forma, a prática consistia em explicar o procedimento aos pacientes com o intuito de informá-los e tranquilizálos, demonstrar interesse na fala deles, conversar, por vezes puxar conversa sobre coisas do cotidiano; assim, a partir de uma postura acolhedora da minha parte em considerá-los como sujeitos, e não objetos da intervenção, considerando o seu contexto de vida e acolhendo o seu sofrimento, comecei a perceber que iam ficando mais tranquilos, ao mesmo tempo que eu já iniciara uma assepsia do local para punção venosa periférica, dando sequência ao procedimento. Com isso, em inúmeras vezes, para eles, o momento era rápido e indolor.

Os usuários chegam ao ambulatório assustados por tudo que é falado nas redes sociais, na televisão e em outros meios de comunicação disponíveis, fora os relatos de experiências de pessoas que também já se submeteram a esse tratamento; e tudo isso concorda para que eles cheguem tensos e receosos de tudo o que aquele momento possa lhes proporcionar (Benjamin, 1987; INCA, 2015).

A partir de uma observação mais apurada e intencionada dos pacientes, fui percebendo que, à medida que eu e outros profissionais de enfermagem adotávamos uma postura acolhedora, ainda que por vezes demorássemos um pouco mais de tempo, mesmo se o investimento nem sempre fosse compreendido, fazia toda a diferença para o êxito no procedimento. Então, percebi-me exercitando o que poderia considerar de 'boa prática de enfermagem'. 
Depreendemos desta experiência que, a partir da adoção das tecnologias leves, entre as quais, bons diálogos com os pacientes, estes deslocam um pouco o foco do procedimento da punção venosa periférica fazendo com que ele seja menos doloroso.

Um efeito que atribuo à pandemia é que ela aproximou, posso dizer, que ajudou a melhorar a comunicação com os pacientes. Essa atitude de se interessar mais pelo outro, pelo que ele estava sentindo, como ele estava naquele momento foi essencial para diminuir a tensão que chegavam à sala de quimioterapia. Assim, esses encontros passaram ser considerados por nós, profissionais de enfermagem, momentos de descontração, pois todos estávamos tensos em virtude da pandemia da covid19.

Esta experiência, que parece pouco complexa, demonstra o quanto à adoção das tecnologias leves, também conhecidas como relacionais fazem toda a diferença na atenção à saúde. A partir do interesse do profissional pelo paciente, podem emergir práticas transformadoras no cuidado em saúde (Merhy \& Franco, 2003).

\subsection{A adoção das tecnologias leves no cotidiano: boas práticas de enfermagem}

Um dos principais dispositivos das tecnologias leves consiste na escuta, na observação, na palavra. Dessa forma, pude perceber como conversar se constitui uma ferramenta que dispara muitas emoções, à serviço do cuidado. As tecnologias leves valorizam os "atos em fala", bem como potencializa o encontro entre os pacientes e os profissionais, a escuta de tudo o que esses sujeitos trazem, com toda sua singularidade (Silva \& Veras, 2016).

Outrossim, à medida que forem implementadas nos serviços, teremos cada vez mais profissionais acolhedores, interessados na vida e atenciosos em ouvir as queixas ou dúvidas dos pacientes. Quando isso acontece, podemos dizer que tais práticas são pautadas no vínculo; e este só é possível ser trabalhado a partir do encontro (Franco, 2016; Merhy et al, 2020;).

Um ponto de partida para a adoção das tecnologias relacionais com os pacientes que fazem tratamento no referido ambulatório reside em reconhecer que, apesar dos esforços da equipe, estar nesse lugar não é confortável, por isso a necessidade de uma equipe saber acolher, ouvir e se mostrar disposta a ajudá-los a enfrentar o cotidiano na sala de quimioterapia. Assim, quando isso emergiu, foi um divisor de águas no meu trabalho (Duarte et al, 2017; Silva et al 2018;).

As tecnologias leves compreendem todos os recursos utilizados para favorecer o encontro, a escuta, o reconhecimento, a porosidade e conhecimentos produzidos na experiência e que são gerenciados pelo encontro. Esses recursos estão em nós; e podemos ativá-los para a implementação de boas práticas. Desse modo, para melhor cuidar do outro, precisamos deixá-los vir à superfície. Este relato possibilita perceber que, ao refletir sobre o processo de trabalho, é possível construir conhecimento para a produção do cuidado (Brasil, 2014; Diniz, 2017; Almeida \& Merhy, 2020).

Com o auxílio da equipe, ajuda e apoio familiar e tratamento psicológico, portanto, essa passagem pode ser amenizada e até mesmo imbuída de afetos, vínculos que aí se estabelecem. Porém, nem sempre os profissionais estão dispostos ou mesmo preparados para desenvolver um bom acolhimento, e isso é um impeditivo para que, de fato, estabeleça-se o acolhimento eficaz nos serviços. É preciso que não somente as práticas profissionais precisam serem postas em análise, mas também as nossas diversas maneiras de agir nesses encontros' a fim de promoverem saúde do usuário sempre (Malta et al, 2016; Malta et al, 2018; Mendes et al, 2016; Norões et al, 2018).

\section{Conclusão}

A associação da adoção das tecnologias leves pelos enfermeiros, as quais consideramos boas práticas, a um cuidado de qualidade confere à enfermagem um lugar diferenciado na atenção à saúde, o que corrobora para a promoção de saúde que prevê, entre outras coisas, a disseminação de práticas inovadoras e efetivas na enfermagem. 
Faz-se necessário incentivar adoção das tecnologias leves pela equipe de enfermagem, principalmente, antes e durante procedimentos dolorosos, invasivos como é o caso da punção venosa periférica. Investir um pouco mais no cuidado para que este seja de fato acolhedor faz toda diferença na qualidade e eficiência do procedimento; e, consequentemente, na resolutividade e melhor qualidade no atendimento ao paciente. Espera-se, com o compartilhamento desta narrativa, a qual ressalto como uma boa prática de enfermagem boa prática, contribuir para um melhoramento no cuidado ao paciente, promovendo uma atenção à saúde organizada a partir do acolhimento.

Para a adoção de boas práticas de enfermagem, principalmente, em setores que o encontro com a morte, com as questões trazidas pela situação de finitude e pelos cuidados paliativos ronda o cotidiano dos profissionais, é preciso haver disponibilidade do profissional em adotar uma postura de acolhimento, em desenvolver ações promotoras de vínculo para a implementação do cuidado. Para tanto, noções e modos de acolher e vincular-se ao outro não são passíveis de serem aprendidas nos livros, tornando-se um grande desafio para os processos de formação no campo da saúde. Sendo uma das proposições a inserção dos alunos de graduação nos serviços cada vez mais precoce, oportunizando um aprendizado em ato, no cotidiano das unidades de saúde, onde os processos de cuidar acontecem.

\section{Referências}

Almeida, S. A., \& Merhy, E. E. (2020). Micropolítica do trabalho vivo em saúde mental: composição por uma ética antimanicomial em ato. Revista Psicologia Política, 20(47),65-75. http://pepsic.bvsalud.org/scielo.php?script=sci_arttext\&pid=S1519-549X2020000100006\&lng=pt\&tlng=pt.

Benjamin W. (1987) Obras Escolhidas - Magia e técnica, arte e política. (3a ed.), Editora Brasiliense; 1 v.

Bondía J. L. (2002) Notas sobre a experiência e o saber da experiência. Rev Bras. Educ.; 19:20-28. • https://doi.org/10.1590/S1413-24782002000100003

Brasil. Ministério da Saúde. (2020) Painel de casos de doença pelo coronavírus 2019 (COVID-19) no Brasil. Ministério da Saúde; https://covid.saude.gov.br/

Brasil. Ministério da Saúde. (2020) Secretaria de Vigilância em Saúde. Doença pelo coronavírus 2019: ampliação da vigilância, medidas não farmacológicas e descentralização do diagnóstico laboratorial. Boletim Epidemiológico Especial (32). https://www.gov.br/saude/pt-br/coronavirus/boletinsepidemiologicos/boletim-epidemiologico-covid-19-no-32.pdf/view

Brasil. (2014) Portaria MS/GM n. ${ }^{\circ}$ 2.446, de 11 de novembro. Redefine a Política Nacional de Promoção da Saúde (PNPS). http://www.anamt.org.br/site/upload_arquivos/legislacao_-_leis_2014_131120141142417055475.pdf

Diniz, A. M. (2017) "Projeto Terapêutico Singular na atenção à saúde mental: tecnologias para o sujeito em crise." SANARE Revista de Políticas Públicas 16.1.https://sanare.emnuvens.com.br/sanare/article/view/1088

Duarte, L. P. D. A., Moreira, D. D. J., Duarte, E. B., Feitosa, A. N. D. C., \& Oliveira, A. M. D. (2017). Contribuição da escuta qualificada para a integralidade na atenção primária. http://gestaoesaude.unb.br http://diadorim.ibict.br/handle1/60http://www.repositorio.u fc.b r/handle /riu fc /31802

Franco, T. (2006). As Redes na Micropolítica do Processo de Trabalho in Gestão em Redes: práticas de avaliação, formação e participação na saúde. Rio de Janeiro: CEPESCIMS/UERJ-ABRASCO.

Huang, C., Wang, Y., Li, X., Ren, L., Zhao, J., \& Hu, Y., et al. (2020) Clinical features of patients infected with 2019 novel coronavirus in Wuhan, China. Lancet.;395:497-506

Instituto Nacional de Câncer José Alencar Gomes da Silva. (2015) Manual de Boas Práticas: Exposição ao Risco Químico na Central de Quimioterapia: Conceitos e Deveres. Inca.

Malta, D. C. et al. (2016) Política Nacional de Promoção da Saúde (PNPS): capítulos de uma caminhada ainda em construção. Ciência \& Saúde Coletiva. 21, 1683-1694.

Malta, D. C. et al. (2016) O SUS e a Política Nacional de Promoção da Saúde: perspectiva resultados, avanços e desafios em tempos de crise. Ciência \& Saúde Coletiva, 23, 1799-1809.

Mendes, R., Pezzato, L. M. \& Sacardo, D. P. (2016) Pesquisa-intervenção em promoção da saúde: desafios metodológicos de pesquisar “com”. Ciência \& Saúde Coletiva 21, 1737-1746.

http://www.cienciaesaudecoletiva.com.br/artigos/a-pesquisaintervencao-em-promocao-da-saude-desafios-metodologicos-de-pesquisar-com/15556?id=15556

Merhy E. E., \& Franco T. B. (2003) Por uma Composição Técnica do Trabalho em saúde centrada no campo relacional e nas tecnologias leves. Apontando mudanças para os modelos tecno-assistenciais. Saúde Debate. 27(65):316-323 
Research, Society and Development, v. 10, n. 9, e15310917806, 2021

(CC BY 4.0) | ISSN 2525-3409 | DOI: http://dx.doi.org/10.33448/rsd-v10i9.17806

Merhy, E. E., \& Franco, T. B. (2003). Por uma Composição Técnica do Trabalho em saúde centrada no campo relacional e nas tecnologias leves. Apontando mudanças paraosmodelostecno-assistenciais. 1. Saúde Pública, Periódico. I. Centro Brasileiro de Estudos de Saúde, CEBES CDD 362.1, 27(65), 316-323. https://doi.org/10.1590/0103-11042019S606

Norões Mota, D. de et al. (2018) Tecnologias da informação e comunicação: influências no trabalho da estratégia Saúde da Família. Journal of Health Informatics, 10. http://www.jhi-sbis.saude.ws/ojs-jhi/index.php/jhi-sbis/article/view/563

Silva, D. N., \& Veras V. (2016). Da teoria dos atos de fala à nova pragmática: os legados de John L. Austin e Kanavillil Rajagopalan. DELTA;32(3):5-19.

Alves da Silva, M. T., Barbosa da Silva Júnior, V., de Oliveira Mangueira, J., \& Lopes de Melo, A. P. (2018). Implantar o acolhimento na estratégia de saúde da família é viável? Revista Em Extensão, 17(1), 153-165. https://doi.org/10.14393/REE-v17n12018-rel05

Souza, H. P., \& Souza, J. R. (2017). Percepção do paciente sobre o protocolo de acolhimento realizado no serviço de oncologia do Hospital Universitário de Brasília (HUB). Tempus - Actas De Saúde Coletiva, 11(1), 187-205. https://doi.org/10.18569/tempus.v11i1.2226 